\title{
Biliary reconstruction in liver transplant patients with primary sclerosing cholangitis, duct-to-duct or Roux-en-Y?
}

\author{
Alireza Shamsaeefar $^{1}$ | Mohammad Shafiee $^{1}$ | Saman Nikeghbalian ${ }^{1}$ \\ Kourosh Kazemi $^{1}$ | Mohsenreza Mansorian ${ }^{1}$ | Nasrin Motazedian ${ }^{2}$ (D) \\ Farsad Afshinnia $^{3}$ |Bita Geramizadeh ${ }^{2}$ | Seyed Ali Malekhosseini ${ }^{1}$
}

\author{
${ }^{1}$ Shiraz Organ Transplant Center, Shiraz \\ University of Medical Sciences, Shiraz, Iran \\ ${ }^{2}$ Shiraz Transplant Research Center, Shiraz \\ University of Medical Sciences, Shiraz, Iran \\ ${ }^{3}$ Division of Nephrology, Department of \\ Internal Medicine, University of Michigan, Ann \\ Arbor, Michigan

\section{Correspondence} \\ Nasrin Motazedian, Shiraz Transplant Research \\ Center, Shiraz University of Medical Sciences, \\ Shiraz, Iran. \\ Email: motazediann@yahoo.com
}

\begin{abstract}
Introduction: Roux-en-Y choledochojejunostomy and duct-to-duct (D-D) anastomosis are biliary reconstruction methods for liver transplantation. However, there is a controversy over which method produces better results. We have compared the outcome of $\mathrm{D}$-D anastomosis vs. Roux-en-Y hepaticojejunostomy in patients with primary sclerosing cholangitis who had undergone liver transplant in Shiraz Organ Transplant Center.

Materials: The medical records of 405 patients with primary sclerosing cholangitis (PSC) who had undergone liver transplant from 1996 to 2015 were reviewed. Patients were divided into two groups: Roux-en-Y group and D-D group. Morbidity, disease recurrence, and graft and patient survival rates were compared between the two groups.

Results: Total of 143 patients underwent a D-D biliary reconstruction, and 260 patients had a Roux-en-Y loop. Biliary complication involved $4.2 \%$ of patients from the D-D group, and 3.9\% from the Roux-en-Y group ( $P=$. 863). Actuarial 1-, 3-, and 5-year patient survival for D-D and Roux-en-Y group was $92 \%, 85 \%$, and $74 \%$; and $87 \%, 83 \%$, and $79 \%$, respectively $(P=.384)$. The corresponding $1-, 3-$, and 5 -year probability of biliary complication was $97 \%, 95 \%$, and 92\%; and 98\%, 97\%, and 94\%, respectively $(P=.61)$.

Conclusion: Duct-to-duct biliary reconstruction in liver transplantation for selected patients with PSC is a good alternative instead of Roux-en-Y biliary reconstruction.
\end{abstract}

KEYWORDS

duct-to-duct, primary sclerosing cholangitis, Roux-en-Y, transplantation

\section{1 | INTRODUCTION}

Primary sclerosing cholangitis (PSC) is a chronic liver disease of unknown causes, which progresses toward fibrosis and biliary obstruction. Eventually, the disease leads to liver failure, cholangitis, or even cholangiocarcinoma (CCA). ${ }^{1}$

To this day, no certain treatment has been identified; the only effective treatment for patient with PSC in the final stage is liver transplantation. ${ }^{2}$ Although liver transplant is an acceptable treatment for patient with PSC in the final stage, biliary complications are more common in this group compared to other groups receiving liver transplants. $^{3}$

Even though the standard method is controversial, a variety of biliary reconstruction methods have been used in liver transplantation for primary sclerosing cholangitis. It was previously reported that a Rouxen-Y loop reconstruction reduces postoperative strictures and has 
better patient and graft survival rate comparing to duct-to-duct (D-D) reconstruction. However, sometimes it is preferable to use D-D anastomosis because of easier accessibility to bile ducts; this method also keeps the anatomy of the biliary system and the sphincter function natural and therefore prevents infection and septicemia. Compared to Roux-en-Y, D-D reconstruction has shorter surgical duration and a more convenient postoperative recovery period. ${ }^{4}$

In a systematic review, one study compared the two methods for anastomosis of the bile ducts (Roux-en-Y vs D-D) in patients with PSC. However, they found no significant differences between the two groups regarding 1-year patient and graft survival rates, risk of biliary complications, and disease recurrence. ${ }^{5}$

One meta-analysis, which analyzed 10 articles relating Rouxen-Y vs D-D methods for reconstruction of biliary ducts in patients with PSC, found no differences between the two techniques regarding occurrence of biliary stricture and biliary anastomotic leakage. Furthermore, there were no differences in postoperative mortality rates. Even though Roux-en-Y has a higher rate of cholangitis, the 1-year graft survival, disease recurrence, and spread of cholangiocarcinoma are comparable between the two groups. ${ }^{6}$

In general, D-D is the preferable method for anastomosis of bile ducts following liver transplantation, but some centers use the Rouxen-Y method in PSC cases. Although some centers have presented satisfactory short-term results from $D-D$ anastomosis of selected patients with PSC, the long-term results of these techniques have not yet been properly explained.

This study aims to compare patient and graft survival rates and biliary complications between the two methods of DD and RY reconstruction of bile ducts in the patients with PSC who visited Nemazee Hospital in Shiraz for liver transplantation during the course of the past 19 years.

\section{2 | MATERIALS AND METHODS}

In this retrospective cohort study, we reviewed the medical records of patients with PSC who underwent liver transplantation in Nemazee Hospital in Shiraz, during the years 1996-2015. The diagnosis of PSC was based on pre-operative magnetic resonance cholangiopancreatography (MRCP) and liver biopsy. A total 405 patients with primary sclerosing cholangitis were enrolled for the final analysis. We made a comparison of patient and graft survival rates and occurrence of biliary complications among them regarding the two methods of biliary duct reconstruction: Roux-en-Y and D-D.

Data collection form consisted of demographic information (age, gender), biliary complications, biliary reconstruction technique, and type of liver transplant, surgical variables and the demographic information of liver donors. Any biliary complications that required hospitalization and involved surgery and endoscopy were considered as postoperative biliary complication. Biliary complications such as leakage, stricture, and residual stones; malignancy in bile duct; and graft loss; and patient survival rates were compared between the two groups.
In our center, decision on the type of biliary reconstruction (D-D anastomosis or Roux-en-Y hepaticojejunostomy) is based on the assessments made by the surgeon. Assessment was based on finding during operation such as normal mucosa and open common bile ducts, without inflammation and wall thickness, and progressive passing the probe into the duodenum. If the surgeon does not find any evidence of impaired bile flow, irregularity of bile duct, malignancy, or pre-malignancy, D-D anastomosis should be the first choice. At least two surgeons make decision for type of biliary construction. This is according to Shiraz transplant center protocol. We take frozen section in cases of malignancy to confirm, RO resection, and also common bile duct completely removed until its entrance to pancreas during Roux-en-Y hepaticojejunostomy, and send for pathology.

\section{1 | Statistical analysis}

For data analysis, in addition to descriptive statistics, we used the $t$ test to compare mean of continuous variables and the chi-square to compare the categorical variables between the two groups with the alpha level of lower than 5\%; patient and graft survival rates were calculated and compared through the log-rank test and the Kaplan-Meier estimator. SPSS-18 was the software used.

\section{3 | RESULTS}

Of the 405 patients who received liver transplants for PSC, 257 (63.5\%) were males; average age of the recipients was $35.1 \pm 11.4$ years. There was a donor/recipient sex mismatch in $44.4 \%$ of the cases. In $93.4 \%$ of all the cases, a whole organ graft was used. Follow-up duration varied from 6 months to 19 years. Two hundred and sixty patients received a Roux-en-Y hepaticojejunostomy anastomosis during liver transplantation, and D-D biliary anastomosis was used for 143 patients.

There was a significant difference between the groups regarding age and gender of donors and recipients, which was clinically insignificant. No significant differences were observed between surgical variables based on the methods of biliary reconstruction (Table 1).

Mean and median survival rates were $132 \pm 4.9$ and 172.5 months, respectively. One-year patient survival was $89 \%$, 3-year survival $84 \%$, and 5 -year survival $77 \%$, for all patients. One-year graft survival was $97 \%$, 3-year $92 \%$, and 5 -year $91 \%$ for all patients. Survival probabilities and events of death were compared between the two groups of $D-D$ and $R-Y$ via the log-rank test.

The D-D group had a 1-year survival of $92 \%$, a 3-year survival of $85 \%$, and a 5 -year survival of $74 \%$, while in the Roux-en-Y group, 1-year survival was $87 \%$, 3-year survival $83 \%$, and 5 -year survival $79 \%$, a result which revealed no significant differences between the two groups $(P$ value $=.384)$ (Figure 1$)$. Seventy deaths occurred after liver transplantation (17.3\%); the causes of death are summarized in Table 2. The most common cause was sepsis (4\%), and chronic rejection was second at $3 \%$. Cholangiocarcinoma was reported in $17(4.2 \%)$ cases. 
TABLE 1 Donor and recipient characteristics and surgical variables

\begin{tabular}{|c|c|c|c|}
\hline & $\begin{array}{l}\text { Duct-to-Duct } \\
\text { Group } 143(35.5 \%)\end{array}$ & $\begin{array}{l}\text { Roux-en-Y } \\
\text { Group } 260 \text { (64.5\%) }\end{array}$ & $P$ Value \\
\hline \multicolumn{4}{|l|}{ Donor variables } \\
\hline Age $(y)^{*}$ & $32.3 \pm 13.3$ & $29.4 \pm 11.3$ & .027 \\
\hline \multicolumn{4}{|l|}{$\operatorname{Sex}(\%)$} \\
\hline Male & $91(65.9)$ & $186(72.7)$ & \multirow[t]{2}{*}{.164} \\
\hline Female & $47(34.1)$ & $70(27.3)$ & \\
\hline \multicolumn{4}{|l|}{ Sex mismatch/match } \\
\hline sex mismatch donor-recipient [n (\%)] & $68(49.3)$ & $107(41.8)$ & \multirow[t]{2}{*}{.154} \\
\hline sex match donor-recipient [n (\%)] & $70(50.7)$ & $149(58.2)$ & \\
\hline \multicolumn{4}{|l|}{ Recipient variables } \\
\hline Age $(y)^{*}$ & $37.1 \pm 12.4$ & $34.1 \pm 10.6$ & .017 \\
\hline \multicolumn{4}{|l|}{$\operatorname{Sex}[\mathrm{n}(\%)]$} \\
\hline Male & $80(56)$ & $176(67.7)$ & \multirow[t]{2}{*}{.019} \\
\hline Female & $63(44.1)$ & $84(32.3)$ & \\
\hline \multicolumn{4}{|l|}{ Surgical variables } \\
\hline Warm ischemia time (min)* & $43.5 \pm 12.8$ & $45.3 \pm 13.3$ & .212 \\
\hline Cold ischemia time $(\mathrm{h})^{*}$ & $6.9 \pm 2.7$ & $6.8 \pm 2.9$ & .781 \\
\hline \multicolumn{4}{|l|}{ Graft type } \\
\hline Whole organ & $127(94.8)$ & 237 (92.6) & \multirow[t]{3}{*}{.506} \\
\hline Living & $6(4.5)$ & $13(5.1)$ & \\
\hline Split & $1(0.7)$ & $6(2.3)$ & \\
\hline
\end{tabular}

FIGURE 1 Survival for patients who had duct-to-duct (D-D) biliary reconstruction vs patients who had a Roux-en-Y anastomosis

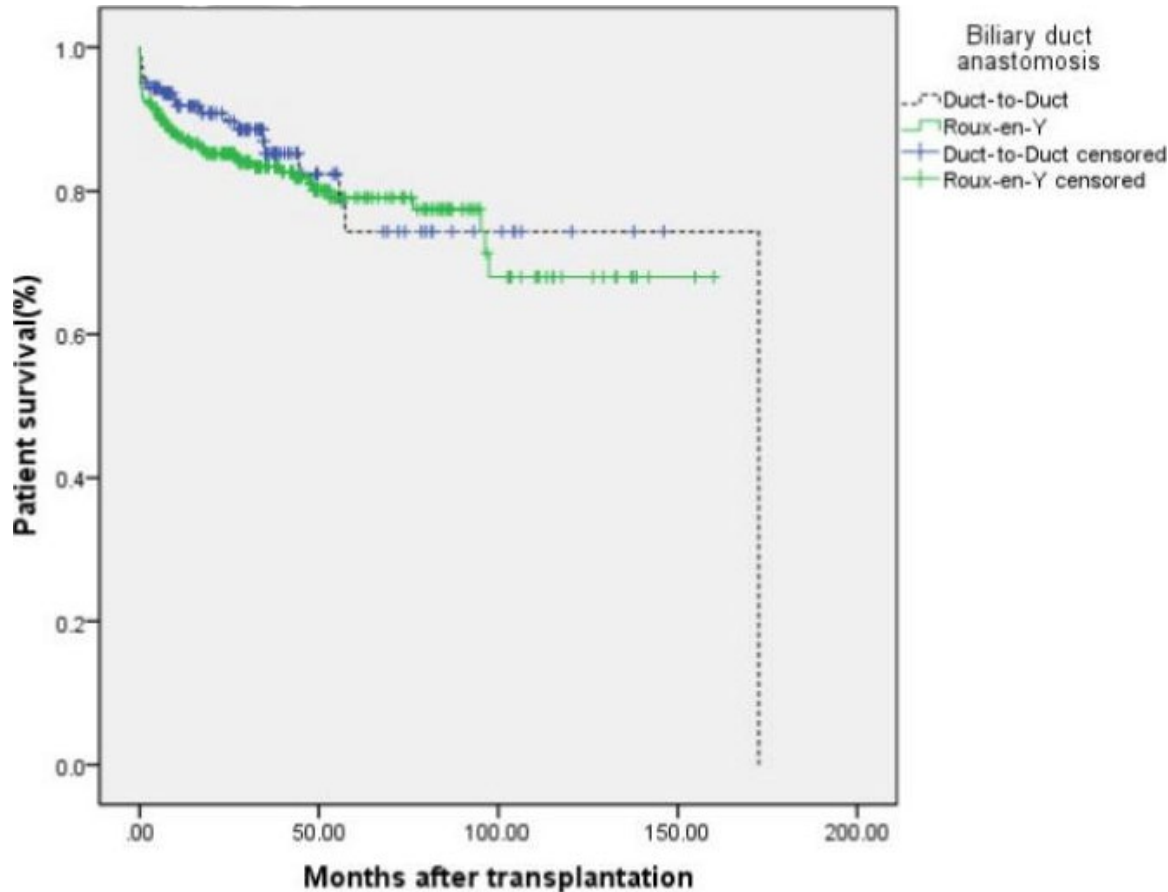

balloon dilation, and stenting were performed for every patient and two cases received common bile duct exploration following PTC. For the complications in the D-D group, ERCP was performed in three cases, Roux-en-Y procedure in two cases and revision of anastomosis in one case. Only one case of cholangitis was reported among the patients in the Roux-en-Y group. 
TABLE 2 Causes of death after liver transplantation

\begin{tabular}{|lll|}
\hline & $\mathbf{n}$ & $\%$ \\
\hline Sepsis/infection & 16 & 4 \\
\hline Chronic rejection & 12 & 3 \\
\hline Cholangiocarcinoma & 10 & 2.5 \\
\hline Graft loss & 6 & 1.5 \\
\hline Malignancy & 5 & 1.2 \\
\hline MI & 4 & 1 \\
\hline PNF & 4 & 1 \\
\hline Severe bleeding & 3 & 0.7 \\
\hline Post-transplantation lymphoproliferative & 2 & 0.5 \\
\hline disorder & & \\
\hline Vascular & 2 & 0.5 \\
\hline Renal failure & 1 & 0.2 \\
\hline
\end{tabular}

The probability over time that a patient transplanted for primary sclerosing cholangitis had biliary complication using the Log-rank test was compared between two mentioned groups.

In the D-D group, 1-year survival was 97\%, 3-year 95\%, and 5-year $92 \%$, respectively; the Roux-en-Y group had a 1-year survival of $98 \%$, a 3 -year $97 \%$, and 5 -year $94 \%$, respectively; as it can be seen, there were no significant differences ( $P$ value $=.61$ ) (Figure 2 ).

From the 405 cases, 35 had graft loss (8.6\%), from which 7 (4.9\%) were in the D-D group and 28 (10.8\%) in the R-Y group. The most common causes of graft failure were PSC recurrence and chronic rejection.

Graft survival and graft loss rates in the two groups were compared via the log-rank test. In the D-D group, 1-year survival was $98 \%$, 3 -year 95\%, and 5-year 92\%; the R-Y group had a 1-year survival of $96 \%$, a 3 -year $91 \%$, and a 5 -year $91 \%$, respectively, which showed no significant difference with the D-D group ( $P$ value=.067; Figure 3 ).
Frequency of liver re-transplantations was not statistically significant between the two groups; the numbers were three $(2.1 \%)$ and four $(1.5 \%)$ in groups D-D and Roux-en-Y, respectively $(P$ value=.702).

\section{4 | DISCUSSION}

Liver transplantation is the only effective treatment available to patients with PSC. Generally speaking, of the two methods of biliary reconstruction, Roux-en- $Y$ has been the method of choice for patients with PSC. This theory of preference is based on using the end section of the recipients' common bile ducts, which could get damaged as a result of chronic PSC. Also, D-D anastomosis increases postoperative biliary complications, even cholangiocarcinoma., ${ }^{7,8}$

In this study, records of PSC (405) cases who received liver transplants in the biggest center in Iran were analyzed; $64.5 \%$ of the patients had received Roux-en-Y anastomosis. The average follow-up was 30 months and 21 days.

Anastomotic strictures (AS) occurred in $4 \%$ of the cases; results showed lower numbers compared to the results from a previous study in America. This difference could have been due to the differences in definition, diagnosis, and follow-up duration. ${ }^{9}$ Studies have reported AS and non-anastomotic stricture (NAS) of $4-9 \%$ and $10 \%-25 \%$, respectively. ${ }^{10,11}$ Incidence rate of AS in our study was comparable with other studies.

Previous studies had not found any differences between these two groups regarding biliary stricture and leakage as our study. ${ }^{8,9}$

This correlation between Roux-en-Y choledochejejunostomy and biliary strictures, which was observed in other studies as well, could be the explanation for its frequent usage; therefore, this method cannot be an independent risk factor for strictures.

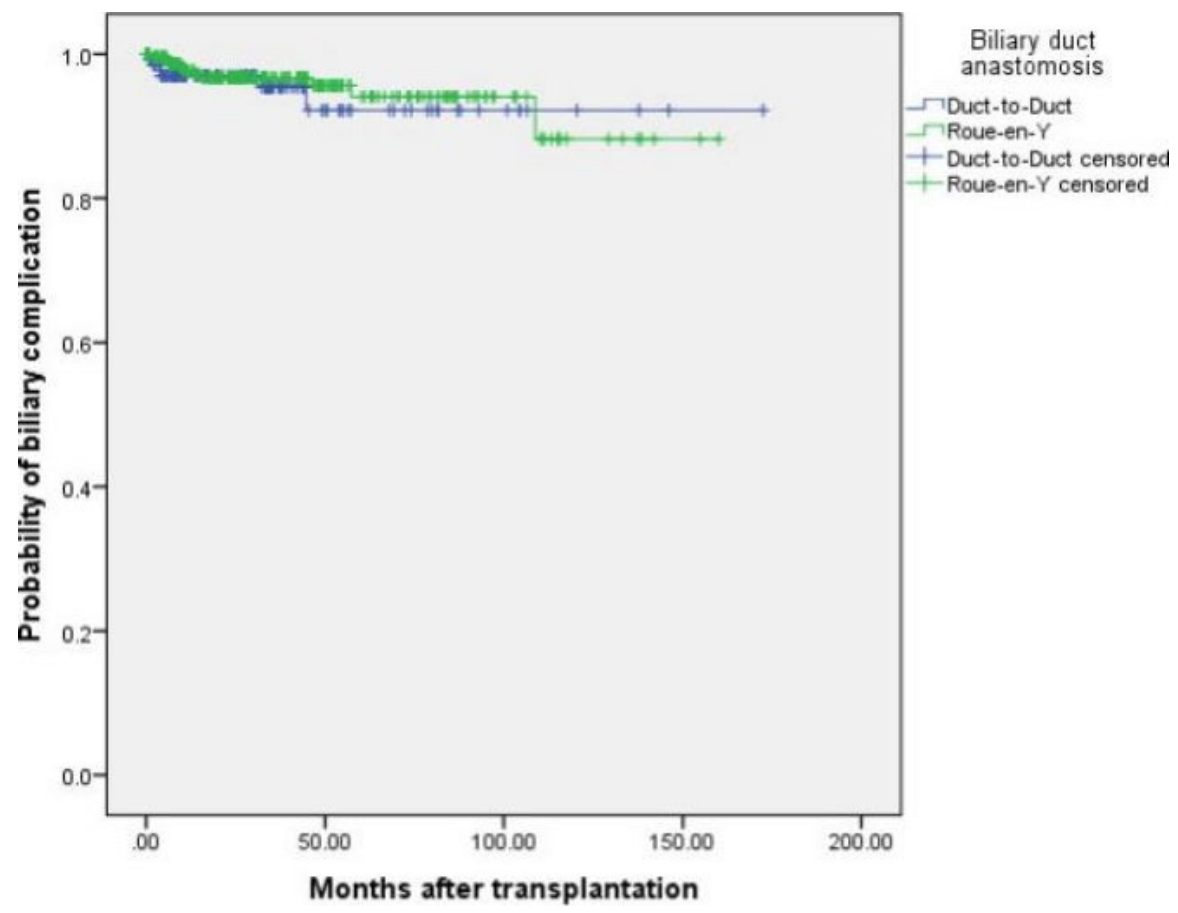

FIGURE 2 The probability of biliary complication in primary sclerosing cholangitis patients after liver transplantation 
FIGURE 3 Graft survival for patients who had duct-to-duct (D-D) biliary reconstruction vs patients who had a Roux-en-Y anastomosis

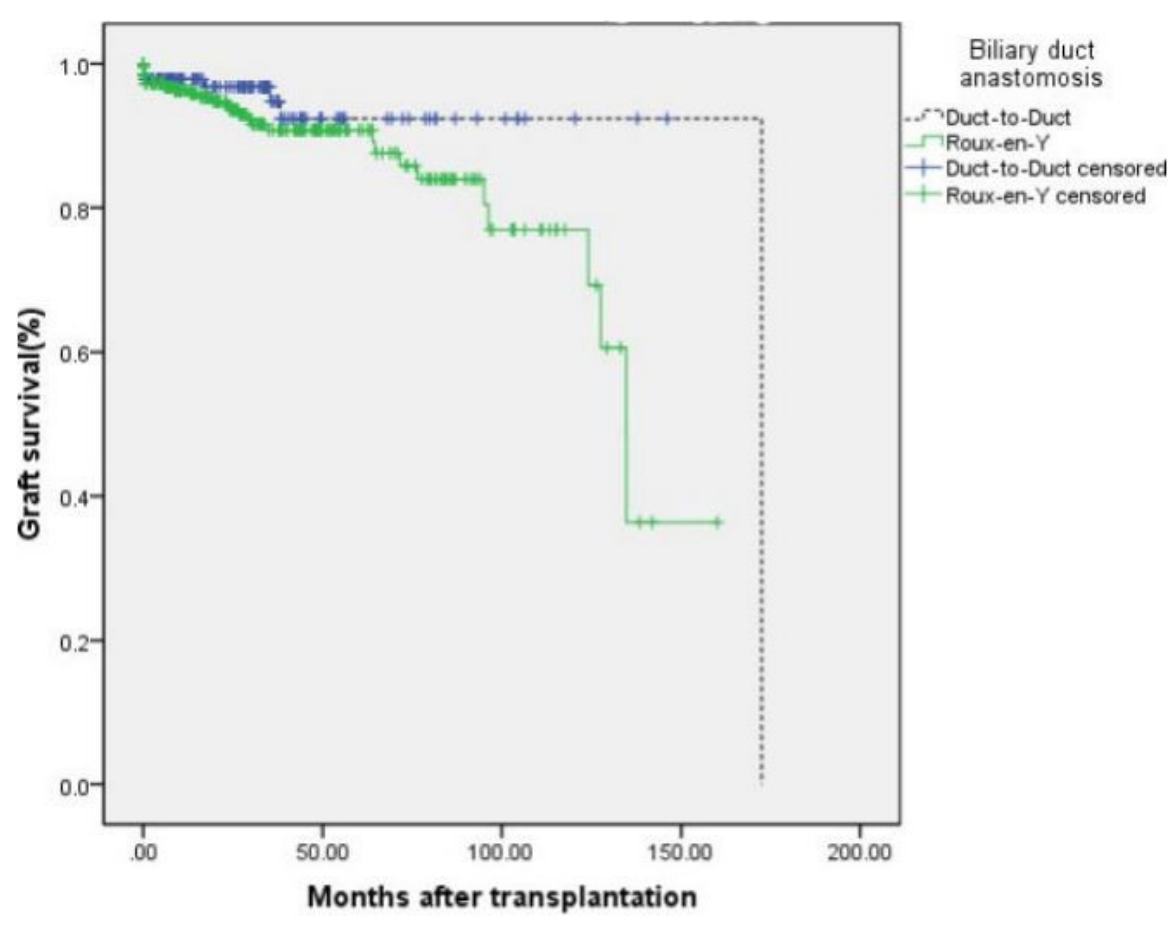

Previous studies have considered Roux-en-Y choledochejejunostomy as a risk factor for NAS, because this method facilitates bacterial ascension through the connection between intrahepatic bile ducts and intestines, and consequently cholangitis. Frequent infections can lead to occurrence and progression of NAS. ${ }^{12}$

Among our patients, only one case of cholangitis was observed in the Roux-en-Y choledochejejunostomy group. One meta-analysis, which made a comparison of the two biliary reconstruction methods, reported higher occurrence rate of cholangitis in the Roux-en-Y group compared to D-D. But there were no differences between the groups regarding strictures in general, AS, and biliary leakage. ${ }^{6}$

In our study, we saw no biliary leakages in any of the reconstructed methods, which was similar to the results of two American studies which used these two methods., ${ }^{3,9}$ However, other studies have reported cases of biliary leakage, but none of those studies had seen any differences between the two reconstructed methods. ${ }^{5}$

Compared to other studies, 5-year survival rate was lower among our patients. ${ }^{12-15}$ But one other study had reported results similar to ours $(76 \%)^{1}$; this could be as a result of the number of accidental cholangiocarcinoma cases observed during surgery.

In this study, we did not observe any differences between the two methods regarding patient and graft survival rates, which was not consistent with results from two meta-analyses. ${ }^{5,6}$ Goss et al. experience showed that PSC patients' survival had no relations with pre-operative factors and biliary reconstruction methods. ${ }^{16}$

In our study, the most common cause of death was sepsis (4\%), a result which was consistent with other studies. ${ }^{15}$

In this retrospective study, we did not have the ability to show the equality of these two methods; it is recommended to design a clinical trial to compare these two biliary reconstruction methods. Despite the fact that the first liver transplantation for patient with PSC was preformed 19 years ago, however, most of the cases received transplants in the recent years and need more time for follow-ups.

\section{5 | CONCLUSION}

MRCP is a good procedure in assessment of patients with PSC before transplant. If the extrahepatic bile ducts are normal and intraoperative observations confirm it, D-D anastomosis would be an acceptable alternative. Furthermore, D-D anastomosis has postoperative advantages such as easier access to bile ducts via endoscopy and lower risk of cholangitis. This method produces similar result to Roux-en-Y choledochojejunostomy with regard to complications and rate of survival.

\section{ACKNOWLEDGEMENT}

The authors would like to thank the Research Consultation Center (RCC) for their invaluable assistance in editing this article. The authors are thankful to Miss Bahareh Khosravi, for her help in data analysis.

\section{CONFLICT OF INTEREST}

None.

\section{REFERENCES}

1. Campsen J, Zimmerman MA, Trotter JF, et al. Clinically recurrent primary sclerosing cholangitis following liver transplantation: a time course. Liver Transpl. 2008;14:181-185.

2. Sutton ME, Bense RD, Lisman T, van der Jagt EJ, van den Berg AP, Porte RJ. Duct-to-duct reconstruction in liver transplantation for 
primary sclerosing cholangitis is associated with fewer biliary complications in comparison with hepaticojejunostomy. Liver Transpl. 2014;20:457-463.

3. Schmitz V, Neumann UP, Puhl G, Tran ZV, Neuhaus P, Langrehr JM. Surgical complications and long-term outcome of different biliary reconstructions in liver transplantation for primary sclerosing cholangitis-choledochoduodenostomy versus choledochojejunostomy. Am J Transplant. 2006;6:379-385.

4. Damrah O, Sharma D, Burroughs A, et al. Duct-to-duct biliary reconstruction in orthotopic liver transplantation for primary sclerosing cholangitis: a viable and safe alternative. Transpl Int. 2012;25:64-68.

5. Wells MM, Croome KP, Boyce E, Chandok N. Roux-en-Y choledochojejunostomy versus duct-to-duct biliary anastomosis in liver transplantation for primary sclerosing cholangitis: a meta-analysis. Transplant Proc. 2013;45:2263-2271. Review.

6. Pandanaboyana S, Bell R, Bartlett AJ, McCall J, Hidalgo E. Metaanalysis of duct-to-duct versus Roux-en-Y biliary reconstruction following liver transplantation for primary sclerosing cholangitis. Transpl Int. 2015;28:485-491.

7. Charatcharoenwitthaya $P$, Lindor KD. Recurrence of primary sclerosing cholangitis: what do we learn from several transplant centers? Liver Transpl. 2008;14:130-132.

8. Al-Judaibi B, Hernandez Alejandro R, Uhanova J, Marotta P, Mosli M, Chandok N. Duct-to-duct biliary anastomosis yields similar outcomes to roux-en-y hepaticojejunostomy in liver transplantation for primary sclerosing cholangitis. Hepat Mon 2015;15:e18811. eCollection 2015.

9. Esfeh JM, Eghtesad B, Hodgkinson P, et al. Duct-to-duct biliary reconstruction in patients with primary sclerosing cholangitis undergoing liver transplantation. HPB (Oxford). 2011;13:651-655.
10. Ryu $\mathrm{CH}$, Lee SK. Biliary strictures after liver transplantation. Gut Liv. 2011;5:133-142.

11. Sharma S, Gurakar A, Jabbour N. Biliary strictures following liver transplantation: past, present and preventive strategies. Liver Transpl. 2008;14:759-769.

12. Hoekstra H, Buis $\mathrm{Cl}$, Verdonk RC, et al. Is Roux-en-Y choledochojejunostomy an independent risk factor for nonanastomotic biliary strictures after liver transplantation? Liver Transpl. 2009;15:924-930.

13. Ponsioen C. Diagnosis, prognosis, and management of primary sclerosing cholangitis. Gastroenterol Hepatol (N Y). 2013;9:453-465.

14. Hildebrand T, Pannicke N, Dechene A, et al. Biliary strictures and recurrence after liver transplantation for primary sclerosing cholangitis: a retrospective multicenter analysis. Liver Transpl. 2016;22:42-52.

15. Graziadei IW, Wiesner RH, Marotta PJ, et al. Long-term results of patients undergoing liver transplantation for primary sclerosing cholangitis. Hepatology. 1999;30:1121-1127.

16. Goss JA, Shackleton CR, Farmer DG, et al. Orthotopic liver transplantation for primary sclerosing cholangitis: a 12-year single center experience. Ann Surg 1997;225:472-481; discussion 481-3.

How to cite this article: Shamsaeefar A, Shafiee M, Nikeghbalian S, et al. Biliary reconstruction in liver transplant patients with primary sclerosing cholangitis, duct-to-duct or Roux-en-Y? Clin Transplant. 2017;31: e12964. https://doi.org/10.1111/ctr.12964 